\title{
Process Maps for Plasma Spray Part II: Deposition and Properties
}

\author{
Xiangyang Jiang, Jiri Matejicek, Anand Kulkarni, Herbert Herman, \\ Sanjay Sampath, Delwyn Gilmore* and Richard Neiser* \\ Center for Thermal Spray Research \\ Department of Materials Science \& Engineering \\ State University of New York at Stony Brook \\ Stony Brook, New York 11794-2275 \\ * Thermal Spray Research Lab, Sandia National Laboratories,

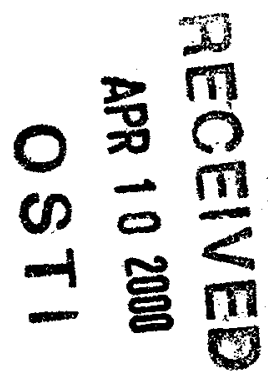

Albuquerque, NM 87185-1130

\begin{abstract}
This is the second paper of a two part series based on an integrated study carried out at the State University of New York at Stony Brook and Sandia National Laboratories. The goal of the study is the fundamental understanding of the plasma-particle interaction, droplet/substrate interaction, deposit formation dynamics and microstructure development as well as the deposit property. The outcome is science-based relationships, which can be used to link processing to performance.

Molybdenum splats and coatings produced at 3 plasma conditions and three substrate temperatures were characterized. It was found that there is a strong mechanical/thermal interaction between droplet and substrate, which builds up the coating/substrate adhesion. Hardness thermal conductivity and modulus increase, while oxygen content and porosity decrease with increasing particle velocity. Increasing deposition temperature resulted in dramatic improvement in coating thermal conductivity and hardness as well as increase in coating oxygen content. Indentation reveals improved fracture resistance for the coatings prepared at higher deposition temperature. Residual stress was significantly affected by deposition temperature, although not significantly by particle energy within the investigated parameter range. Coatings prepared at high deposition temperature with high-energy particles suffered considerably less damage in wear tests. Possible mechanisms behind these changes are discussed within the context of relational maps which are under development.
\end{abstract}

Key words: plasma spray, processing map, molybdenum, microstructure, processing / property relationships

\section{Introduction}

Thermal spraying is a highly complex deposition process with a large number of interrelated variables. Traditionally, satisfactory deposit quality is achieved through a Design of Experiment (DoE) approach. Numerous engineering parameters, such as power level, primary plasma gas type and flow rate, auxiliary plasma gas type and flow rate, stand-off distance, etc. have to be tried empirically and systematically through parameter matrix to find out the optimal parameters. The insight of the intermediate sub-processes and the corresponding mechanisms are largely unknown.

From a scientific point of view, particle velocity, particle temperature, and substrate/deposit temperature $\left(T_{s}\right)$ are the most important parameters which determine the deposit build-up process and deposit properties. Particle characteristics such as temperature and velocity affect almost every single sub-process in the deposition process, such as droplet spreading/solidification, droplet/substrate or previously deposited layer interaction, while substrate temperature has been found to have a significant influence on splat morphology, deposit microstructure and properties [1-3]. With an increase of substrate temperature, splat morphology changes from highly fragmented to a contiguous, disk-like shape [1-3], while deposit integrity and properties are enhanced [3,4].

In this collaborative study, an effort was made to determine relationship between plasma parameters, particle parameters and deposit microstructure/properties. Formation of single splats, as a building block of the entire deposit, is investigated as well. Deposit microstructure buildup and property results are presented in this paper. 


\section{DISCLAIMER}

This report was prepared as an account of work sponsored by an agency of the United States Government. Neither the United States Government nor any agency thereof, nor any of their employees, make any warranty, express or implied, or assumes any legal liability or responsibility for the accuracy, completeness, or usefulness of any information, apparatus, product, or process disclosed, or represents that its use would not infringe privately owned rights. Reference herein to any specific commercial product, process, or service by trade name, trademark, manufacturer, or otherwise does not necessarily constitute or imply its endorsement, recommendation, or favoring by the United States Government or any agency thereof. The views and opinions of authors expressed herein do not necessarily state or reflect those of the United States Government or any agency thereof. 


\section{DISCLAIMER}

Portions of this document may be illegible in electronic image products. Images are produced from the best available original document. 


\section{Experimental}

A plasma-densified spherical molybdenum powder with diameters in the range of 5 to 44 micrometers and a mean size of 30 micrometers, provided by Osram Sylvania Inc. (designation SD 152) were selected. Polished 304 stainless steel substrates (roughness $\mathrm{Ra}<0.1 \mathrm{um}$ ) and grit-blasted mild steel substrates were used for splats collection and coatings respectively.

Processing was carried out at Sandia National Labs with a Miller SG-100 plasma gun under ambient atmosphere $(0.83 \mathrm{~atm})$. Three plasma power / particle energy $(\mathrm{PE})$ levels were used. The corresponding in-flight particle characteristics such as temperature, velocity and size were measured with a Tecnar DPV 2000 particle diagnostic system. Substrates were mounted in a fixture rotating at $240 \mathrm{rpm}, 100 \mathrm{~mm}$ away from the exit of the torch. The surface temperature $\left(T_{s}\right)$ was monitored and controlled by an air cooling feedback system. Splats were collected on substrates nominally at three temperatures: $\operatorname{low}\left(115^{\circ} \mathrm{C}\right)$, medium $\left(325^{\circ} \mathrm{C}\right)$, and high $\left(465^{\circ} \mathrm{C}\right)$. Details of the spray conditions are listed in Table 1.

Table 1. Plasma spray parameters for preparation of splats and coatings

Plasma power/ Current Plasma Gas Carrier Gas Particle Temp. Particle Vel. Target substrate temperature

\begin{tabular}{ccccccc} 
Particle energy & (amps) & Flow (SLPM) & Flow (SLPM) & $\left({ }^{\circ} \mathrm{C}\right)$ & $(\mathrm{m} / \mathrm{s})$ & $\left({ }^{\circ} \mathrm{C}\right)$ \\
\hline Low & 540 & Ar: 50, He: 12 & Ar: 3.0 & 2780 & 130 & $115,325^{*}$ \\
Medium & 700 & Ar: 50, He: 18 & Ar: 2.0 & 2980 & 153 & $115^{*}, 325^{*}$ \\
High & 860 & Ar: 50, He: 26 & Ar: 1.0 & 3120 & 183 & $115^{*}, 325^{*}, 465$
\end{tabular}

SLPM: standard liters per minute. Particle characteristics are experimentally measured means for these conditions.

* Coatings were made at these substrate temperatures $\left(T_{s}\right)$.

Characterization includes the observation and measurement of splats and the corresponding craters formed on the substrates. The procedure is as follows: First markups were made on the substrates by indentation so that the splats can be numbered and located, subsequently splats were observed with optical microscopy and scanning electron microscopy (SEM) and dimensions were measured; following this, the splats were etched away with saturated nitric acid and craters were characterized. The dimensions of splats and craters were measured with a Zygo New View 200 non-contact surface profiler (a scanning white-light interferometer) [5]. Hardness measurement were carried out with a Buehler Micromet II microhardness tester using a Vickers indentor and a $500 \mathrm{~g}$ load on the transverse cross section of the coating. High load indentation was carried out with a Mitutoyo AVK-C2 hardness tester using a $20 \mathrm{~kg}$ load. Thermal conductivity measurements of the coatings were carried out on free-standing cylindrical specimens by a laser flash technique, using a Holometrix Thermal Properties Instrument. Open porosity was measured by the mercury intrusion technique, using a Quantachrome Autoscan 33 porosimeter.

Residual stresses were measured in $0.1 \mathrm{~mm}$ thick coatings on stainless steel. The " $\sin ^{2} \psi$ " X-ray diffraction technique [6] was applied, using a Siemens D500 diffractometer with Ni-filtered Cu radiation, reflection from (321) crystal planes with elastic constants $\mathrm{E}=313 \mathrm{GPa}$ and $\nu=0.31$ and 10 sample tilts from $\psi=-52$ to $52^{\circ}$.

\section{Results and Discussion}

The morphology of splats produced at 7 conditions and related results are presented in a separate paper in this volume [7]. Only significant results are summarized here.

Droplet/substrate interaction and the establishment of adhesion. It was found that molybdenum splat morphology is sensitive to particle energy (PE) and substrate temperature $\left(T_{s}\right)$. At low $T_{s}$, all splats exhibit highly splashed morphology with a fragmented core structure and debris scattered radially. With an increase in substrate temperature and decrease of particle energy, contiguous splat are more readily formed as schematically shown in Fig. 1.

Coating Microstructure and integrity. Figure 2 shows the cross section of coatings prepared at $\mathrm{Ts}=115^{\circ} \mathrm{C}$ at three particle energy conditions. All of them display lamellae-like structure. With the increase of particle energy, the lamellae in the coatings become slightly thinner, due to the higher particle velocity. So the interface number is larger for higher PE for a given thickness. It seems that porosity is slightly higher in the low PE coating. Inter-lamellae pores can be discerned clearly in all three coatings. Coatings produced at higher $\mathrm{T}_{\mathrm{s}}$ show similar structures. 


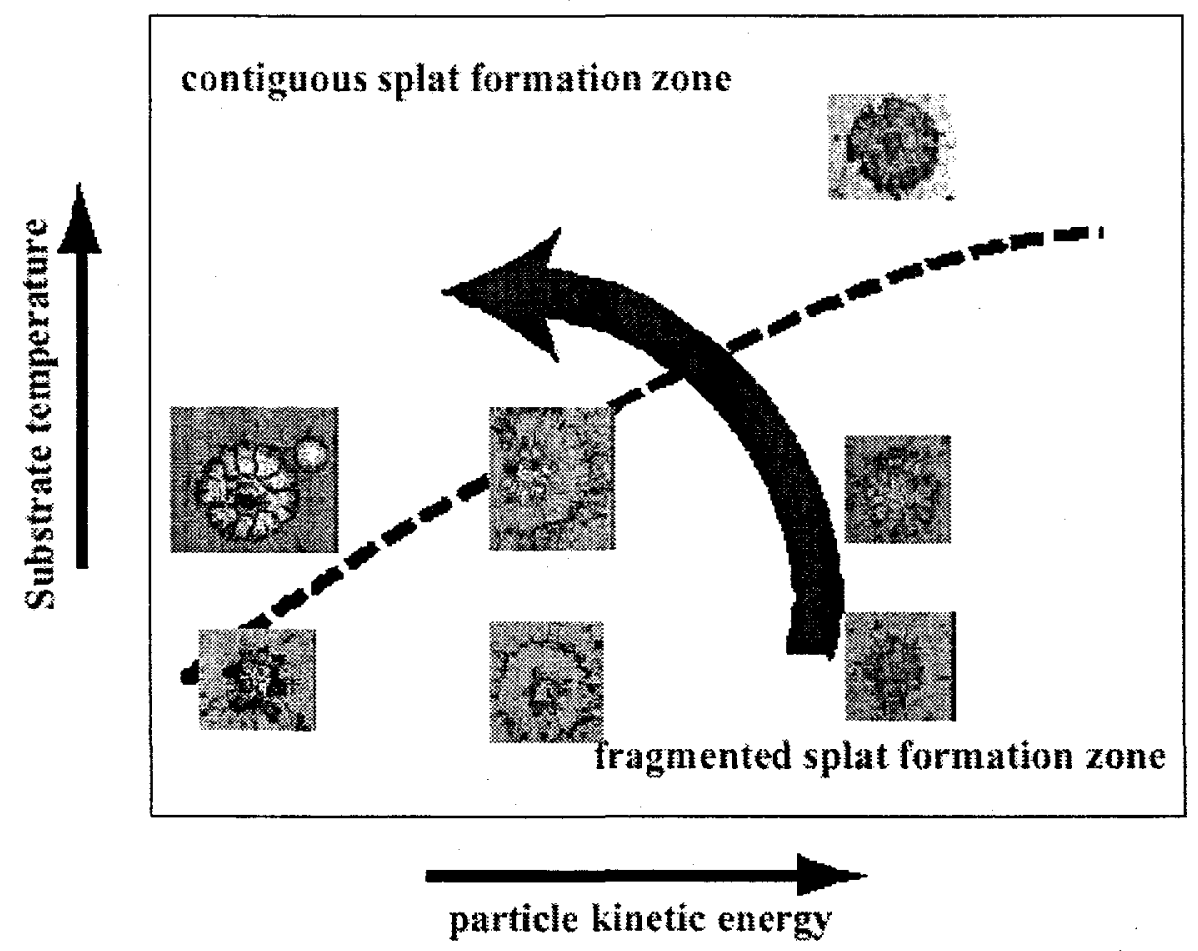

Figare 1 Contiguous splat formation particle energy/substrate temperature zone
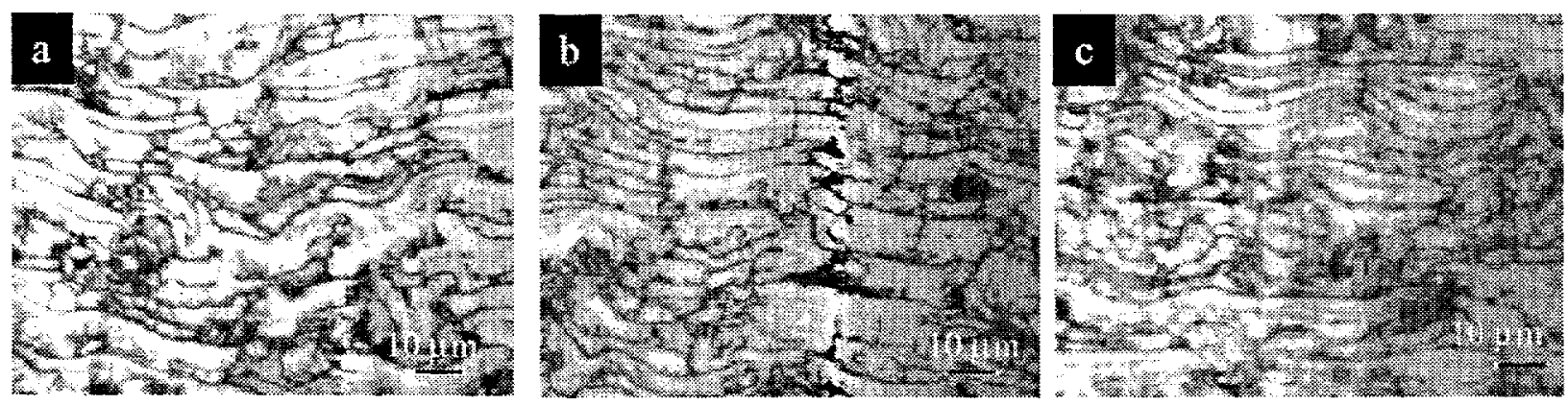

Figare 2 cross section of caling made at three particie energy conditions at $T s=115 \mathrm{C}$. etchant: Muradami's. a: low PE, b: nedium PE, c: high PE

Indentation was used to reveal any possible difference in coating mechanical response originating from different microstructures at various $P E$ and $T_{s}$ conditions. The impressions and surrounding coating structures are shown in Fig. 3. At a load of 500 gram, many inter-lamellar cracks can be found around the impression for all the coatings produced at $T_{s}=115^{\circ} \mathrm{C}$ regardless of the particle energy level. Meanwhile, for the coatings produced at higher $\mathrm{T}_{\mathrm{s}}$, no cracks were observed around the impression. It is noticed that inter-lamellae boundaries are more readily seen in the higher $T_{s}$ coatings. This feature is probably due to the higher oxide level on the interface associated with higher $T_{5}$ coatings (see discussion in the oxidation section). With the increase of load to up to 20 $\mathrm{kg}$, low $T_{s}$ coatings show similar cracking pattern and higher $T_{s}$ coatings show long cracking initiated from the tips of indentation diagonal and propagating parallel to the deposit/substrate interface along lamellae/lamellae boundaries as shown in Fig. 4 (medium PE). 


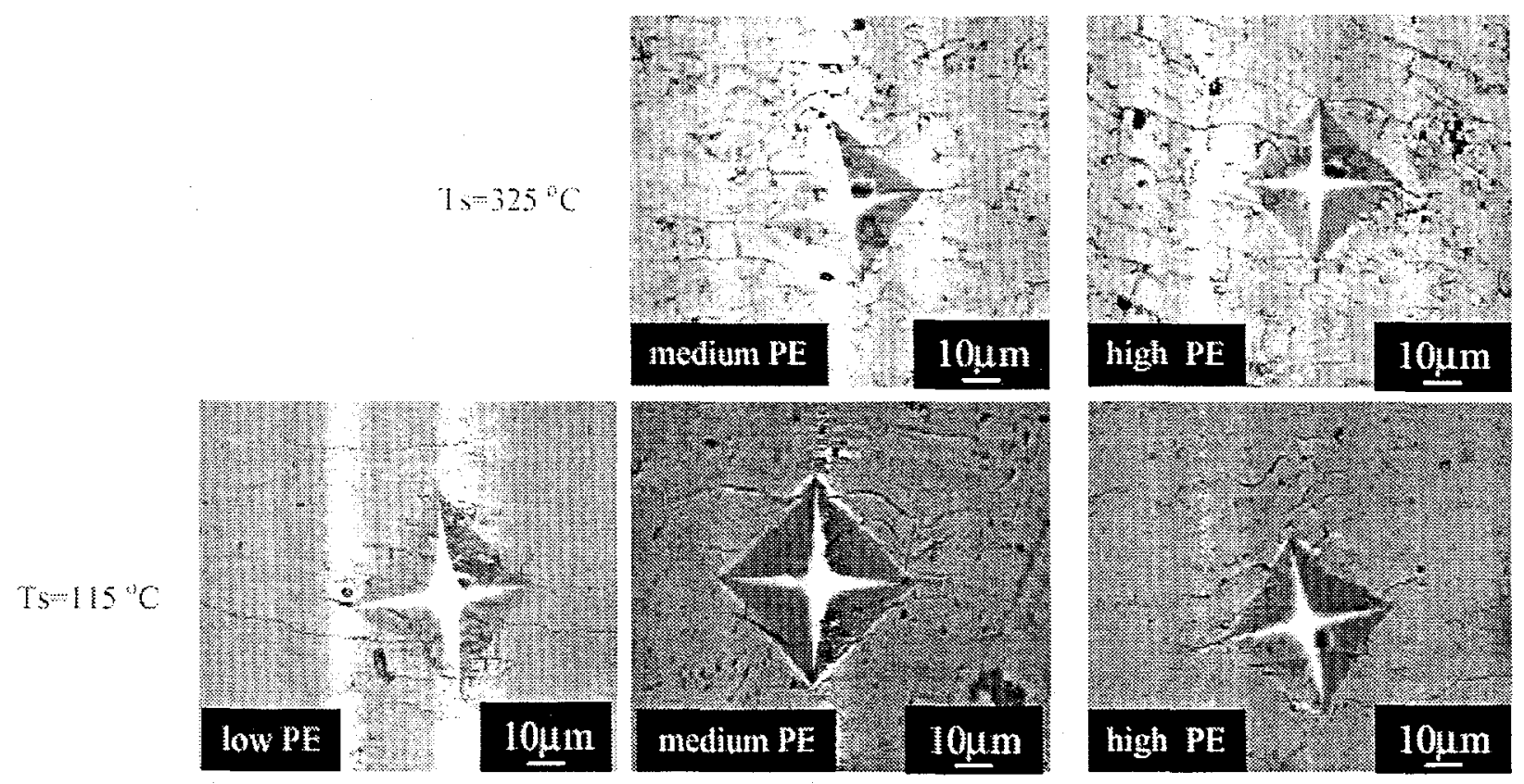

Figure 3 Indentation mark and cracking pattern on caatings produced at different particle cnergy

(P ${ }^{\prime}$ ) and $T$ s enditions, lad $500 \mathrm{~g}$
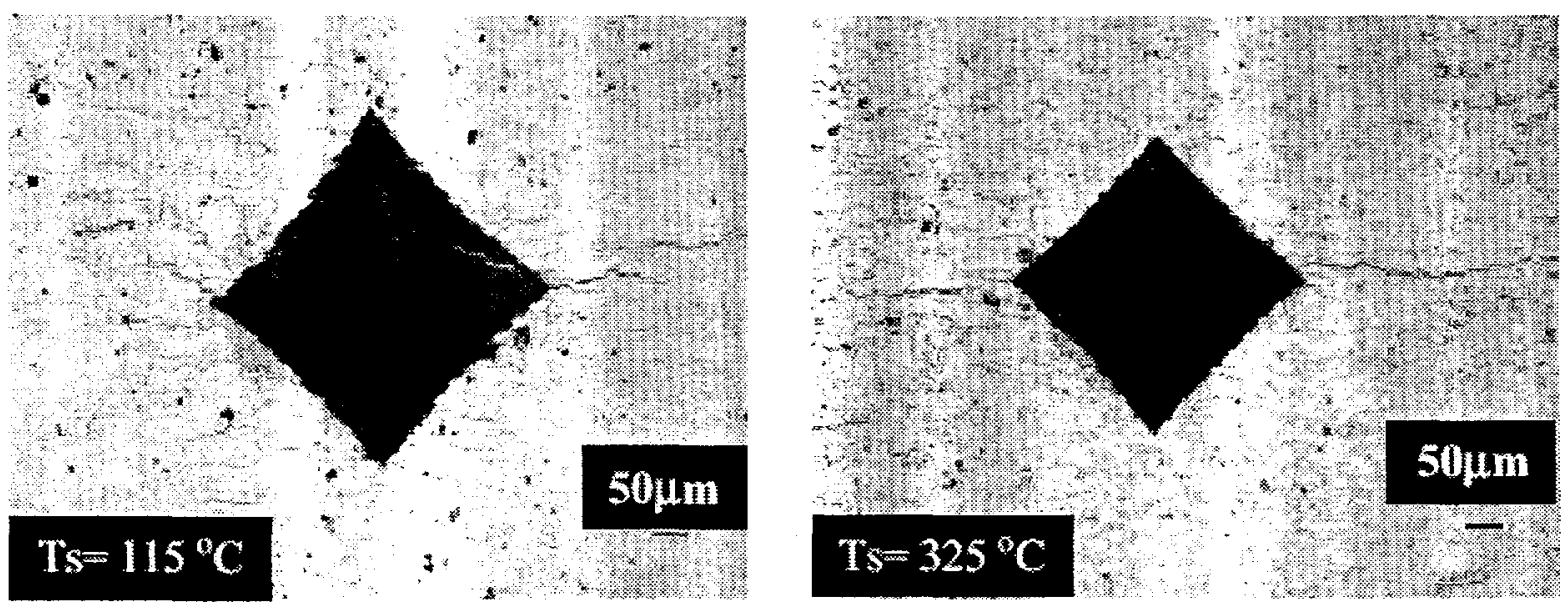

Figure 4 indentation mark and crack pattern on coatings produced at two Ts conditions, particle energy level: medtum; load: $210 \mathrm{~kg}$

Initiation of cracking is determined by the local stress level and the coating fracture toughness. The existence of many short cracks around the indentations on the low Ts coatings indicates that the interlamellar adhesion is much weaker compared with coatings produced at higher $T_{i}$. The stress is relaxed even when it is small and therefore no large stress huilt up near the indentation tip. In the case of higher $T_{s}$ coatings, stress is built-up and cracks initiated at the indentation tip where maximum stress exists. This observation is consistent with our observation before in another study, where high $\mathrm{T}_{\mathrm{s}}$ molybdenum coatings showed trans-lamellae fracture features as compared with an inter-lamellae fracture mode in low $\mathrm{T}$, deposits [3]. The fact that cracks are parallel to the deposit/substrate interface is a manifestation of the anisotropy in deposit structure and properties. 
Residual stress. The effects of deposition temperature and particle energy on residual stress in $0.1 \mathrm{~mm}$ thick coatings are presented in Table 2 and Fig. 5. As can be seen from Fig. 5a, deposition temperature has quite a dramatic effect on the coating residual stress. When the temperature increases from $120^{\circ} \mathrm{C}$ to $280^{\circ} \mathrm{C}$, the stress shifts from tensile to compressive and becomes even more compressive above $300^{\circ} \mathrm{C}$. This trend can be explained by increasing contribution of thermal stress, which is proportional to the difference in coefficients of thermal expansion (CTE) of the substrate and the coating, temperature drop during post-deposition cooling and coating modulus. Since the coating's CTE is lower than the substrate's, the thermal stress is compressive and its magnitude increases with deposition temperature, due to larger temperature drop after the deposition. Increasing modulus with deposition temperature [3] can explain the increase in slope on Fig. 5a. The effect of particle energy is rather negligible, as shown on Fig. 5b. Only a small trend of decreasing stress with particle energy was observed at lower deposition temperature, however, the differences were smaller than experimental error. The particle energy effects may become more significant in thick coatings, where the associated changes in splat/coating formation will affect the microstructure and thus mechanical properties.

Compressive stress in the coating may be beneficial for its mechanical properties, since it would close the existing cracks and suppress crack propagation, but too high magnitude can lead to delamination at the coating/substrate interface. The stress state and mechanical properties can be easily controlled by the deposition temperature and therefore can be tailored to specific application.

Table 2 Residual stresses for coatings produced at various particle energy and substrate temperature levels

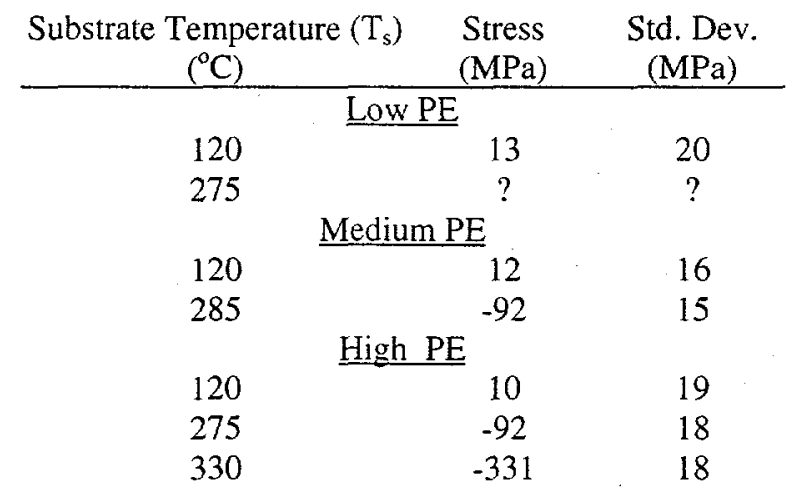
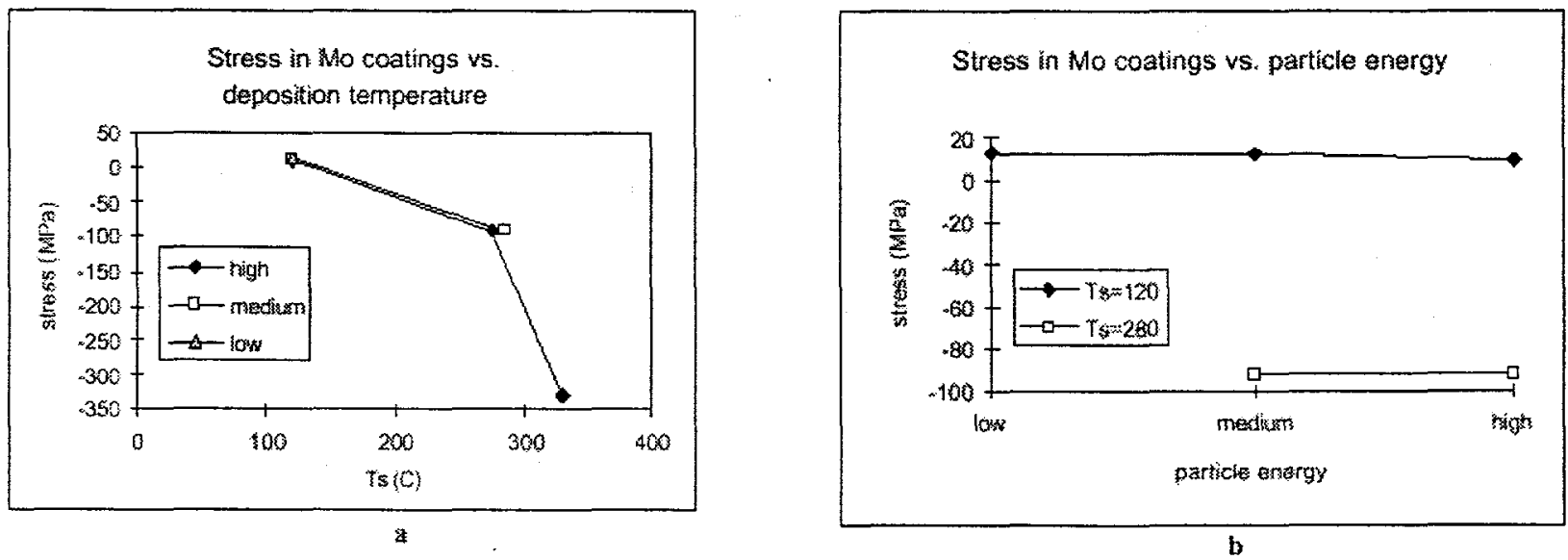

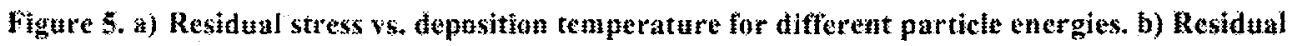
stress vs. particle energy for different deposition temperatares. 


\section{Coating properties}

Oxygen content: The oxygen content of coatings produced at various conditions is plotted in Fig. 6 . It is clear that at $115^{\circ} \mathrm{C}$, with increasing particle velocity, oxygen content decreases. For the same particle velocity, coatings produced at higher $T_{s}$ show higher oxygen content. This result indicates that both in-flight particle oxidation and post-depositing surface oxidation contribute to the overall coating oxidation.

It is shown through modeling that in-flight oxidation is primarily controlled by the residence time of the molten particles in the oxygen containing flame [8]. Since the nozzle/substrate distance is fixed in all the conditions, high velocity particles will have low oxygen content. At higher $T_{s}$, post-depositing surface oxidation is much more significant, so the overall oxygen content is increased. The reason for the higher oxygen content in the high velocity coating as compared with the medium velocity coating at high $T_{5}$ is most likely that there was a slightly higher $T_{s}$ for the $183 \mathrm{~m} / \mathrm{s}$ coating $\left(325^{\circ} \mathrm{C}\right)$ than for the $153 \mathrm{~m} / \mathrm{s}$ coating $\left(310^{\circ} \mathrm{C}\right)$, instead of the nominal $\mathrm{T}_{\mathrm{s}}$ of $325^{\circ} \mathrm{C}$ for both.

Hardness and porosity: As Figs. 7 and 8 show, with the increase of particle kinetic energy (calculated from particle velocity, and size data), coating porosity decreases significantly and coating hardness increases slightly for the two $T_{s}$ investigated. Substrate temperature shows a dramatic effect on coating hardness. With an increase of $T_{s}$ from $115^{\circ} \mathrm{C}$ to $325^{\circ} \mathrm{C}$, hardness increases by more than $20 \%$. However, the porosity change is not as dramatic.

Thermal Conductivity: Table 3 lists the thermal conductivity data for coatings made at various conditions. These values are only about 10 to $30 \%$ of that for sintered molybdenum, associated with the existence of many interlamellae interfaces and pores. It appears that thermal conductivity is not significantly affected by particle energy but very sensitive to Ts. Thermal conductivity tripled with the increase of substrate temperature from $115^{\circ} \mathrm{C}$ to $325^{\circ} \mathrm{C}$, despite the fact that the oxygen content increased. Thermal conductivity is an indication of inter-lamellae contact quality. The dramatic increase of thermal conductivity reveals significant enhancement of inter-lamellae bonding and adhesion.

Table 3 Coating Thermal Conductivity

\begin{tabular}{ccc} 
& \multicolumn{2}{c}{ Thermal conductivity (W/m-K) } \\
\cline { 2 - 3 } & $\mathrm{T}_{\mathrm{s}}=115^{\circ} \mathrm{C}$ & $\mathrm{T}_{\mathrm{s}}=325^{\circ} \mathrm{C}$ \\
\cline { 2 - 3 } Medium PE & 13.0 & 46.5 \\
High PE & 16.6 & 45.3
\end{tabular}

Discussion

It is evident that substrate temperature has a far greater effect on coating fracture resistance, hardness, residual stress and inter-lamellae contact than particle energy in the investigated parameter ranges. Coatings produced at higher $T_{s}$ also have lower coefficients of friction and higher scratch resistances [10]. Although increasing particle energy does reduce coating porosity and increase hardness, the improvement is not dramatic.

The mechanism of improving fracture toughness, hardness, inter-lamellae adhesion at higher $T_{\mathrm{s}}$ is not clear yet. Our current understanding suggests that there are three possibilities: 1 ) less trapped air in the pores due to lower air density at higher temperature; 2) lower air density results in less severe disturbance to droplet spreading, which may lead to better contact; 3) a thicker splat surface oxide layer (presumably $\mathrm{MoO}_{3}$ as suggested in the literature [10]) as evidenced by the higher oxygen content may help to improve the contact quality. An XPS study shows traces of $\mathrm{MoO}_{3}$ on the as-sprayed coating surface, but X-ray and electron diffraction of similar coatings did not reveal detectable amount of $\mathrm{MoO}_{3}$ inside the coatings [11]. It could be that the low melting point oxide melts and dissolves into the impinging droplet, help to form good metallurgical bonding between splats and improve thermal conductivity. If the oxide exists as a solid film at the interface, the coating thermal conductivity would be reduced.

Similar coating property enhancement at higher $T_{s}$ was reported in partially stabilized zirconia deposits [4], so mechanism 3 is unlikely to be the only mechanism. In reality, perhaps all three mechanisms are active. In terms of reducing air density, raising substrate temperature in APS is just like spraying in a low pressure environment, which usually leads to higher coating density and enhanced properties. Further investigation is in progress. 


\section{Summary}

It was found that there is a strong mechanical / thermal interaction between droplet and substrate, which builds up the coating/substrate adhesion. Hardnèss, thermal conductivity increase, oxygen content and porosity decreases with increase of particle velocity. Increasing deposition temperature resulted in dramatic improvement in coating thermal conductivity and hardness as well as increase in coating oxygen content. The residual stress in the coatings changes from tensile to compressive as the deposition temperature increases, which makes this parameter an effective means of controlling the stress level. The observed effects of particle energy on coating residual stress levels were small. Indentation reveals improved fracture resistance for the coatings prepared at higher deposition temperature. In the investigated parameter range, substrate temperature has a larger effect on coating property as compared with particle energy.

\section{Acknowledgements}

This work was supported by the MRSEC Program of the National Science Foundation under Award Number DMR-9632570. Sandia is a multi-program laboratory operated by Sandia Corporation, a Lockheed Martin Company. for the United States Department of Energy under Contract DE-AC04-94AL85000.

\section{References}

1. L. Bianchi, F. Blein, P. Lucchese, M. Vardelle, A, Vardelle and P. Fauchais, in: C.Berndt and S. Sampath (Eds.), Thermal Spray Industrial Applications, p.569, ASM International, Metals Park, OH, (1994)

2. M. Fukumoto, S. Katoh, and I. Okane, in: Ohmori, A. (Ed.). Proceeding of the Integrated Thermal Spray Conference, p353, Kobe Japan, May, 1995, Japan High Temperature Society, (1995)

3. X. Jiang, J. Matejicek and S. Sampath, Materials Science \& Engineering A, 272, 189-198 (1999).

4. S. Sampath, X. Jiang, J. Matejicek, Materials Science \& Engineering A, 272, 189-198 (1999).

5. P.De Groot, J. Modern Optics, 42. 389-401 (1995).

6. J. Matejicek, S. Sampath, J. Dubsky, J. Thermal Spray Tech., 7, 489-496, (1998)

7. X. Jiang, Y. Wan, X. Y. Wang, H. Zhang, R. Goswami, H. Herman and S. Sampath, this volume.

8. Y. Wan, X. Jiang, J. Fincke and S. Sampath, this volume.

9. S. De palo, Department of Materials Science \& Engineering, State University of New York at Stony Brook, Stony Brook, NY11794, unpublished work.

10. Mo-O Phase diagram, Bulletin of Alloy Phase Diagrams, 1, 85. (1980)

11. G. Ramasis, H.Herman, S.Sampath, X. Y. Jiang, Y. Tian and H.Halada, Department of Materials Science \& Engineering, State University of New York at Stony Brook, Stony Brook, NY11794 Oxidation of plasma sprayed molybdenum coating, unpublished results. 